\title{
Manejo de pacientes con diagnóstico de adenoma hipofisario productor de prolactina. Experiencia del Hospital San José
}

\section{Diana Cristina Henao ${ }^{1}$ William Rojas ${ }^{2}$}

${ }^{1}$ Médica Internista, Endocrinóloga, Fundación Universitaria de Ciencias de la Salud.

${ }^{2}$ Médico Internista, Endocrinólogo, Profesor asociado Fundación Universitaria de Ciencias de la Salud. Jefe Departamento de Endocrinología, Hospital San José, Bogotá

Dirección de contacto

dchenao@fucsalud.edu.co

\section{Resumen}

Introducción: El prolactinoma es el tumor hipofisiario funcionante más frecuente.

Objetivo: Describir la experiencia del servicio de endocrinología del Hospital San José de Bogotá en el manejo de pacientes con prolactinoma que consultaron entre enero de 2006 y diciembre de 2012.

Métodos: Serie de casos. Se describieron variables demográficas, clínicas, seguimiento radiológico anual, prolactina (PRL) basal, a los 6 y 24 meses. Ingresaron pacientes con adenoma hipofisario documentado por resonancia nuclear magnética (RNM) contrastada, PRL sérica mayor de $100 \mathrm{ng} / \mathrm{ml}$, o diagnóstico extrainstitucional de prolactinoma.

Resultados: Se analizaron 95 pacientes; 71\% con microprolactinomas y $28,4 \%$ con macroprolactinomas. La mediana de duración del tratamiento en pacientes con microprolactinomas fue 73,4 meses con una mediana de dosis acumulada de cabergolina (CAB) de $52 \mathrm{mg}$. En las personas con macroprolactinoma fue de 65 meses, con mediana de dosis acumulada de CAB de 156 mg. El 78,3\% inició tratamiento con bromocriptina (BRC). Ocho pacientes cumplieron criterios de remisión.

Conclusión: La población atendida en el Hospital San José tiene características similares a las registradas en la literatura; sin embargo, el porcentaje de remisión es bajo, lo cual, posiblemente está asociado al uso de bajas dosis de agonistas de dopamina. Se requieren estudios prospectivos para aclarar si la dosis acumulada es un factor predictor para aumentar el porcentaje de pacientes con retiro exitoso y establecer la mejor estrategia para retiro de agonistas de dopamina en pacientes con prolactinomas.

Palabras clave: prolactinoma, Hiperprolactinemia, agonistas de dopamina, cabergolina, bromocriptina.

\section{Summary}

Objective: To describe our experience in the Endocrinology Service of Hospital San José in the treatment of patients with prolactinoma who were seen between 2006 and 2012.

Methodology: Case series. Demographic and clinical variables were described, as well as radiological monitoring once yearly and basal prolactin (PRL) measurements at 6 and 24 months. The patients included suffered from pituitary adenoma documented by contrast magnetic resonance imaging (CMRI), with serum PRL $100 \mu \mathrm{g} / \mathrm{L}$ or above, or who had been diagnosed with prolactinoma by another institution.

Results: 95 patients were analyzed. 71\% presented with microprolactinomas and $28.9 \%$ with macroprolactinomas. The median treatment duration for patients with microprolactinomas was 73.4 months, with a median accumulated dosage of cabergoline (CAB) of $52 \mathrm{mg}$. For macroprolactinomas, the median treatment duration was 65 months and the median accumulated dose of cabergoline was $156 \mathrm{mg} .73 .8 \%$ of patients received bromocriptine. Eight patients met remission criteria.

Conclusion: The patient population treated at Hospital San José has similar features to that described in the literature. However, the remission rate is low, possibly explained by the use of low doses of dopamine agonists. Prospective studies are required to clarify whether the cumulative dose is a predictive factor for increasing the rate of patients with successful withdrawal and to establish the best strategy to withdraw dopamine agonists in patients with prolactinomas.

Key words: Prolactinoma, Hyperprolactinemia, Dopamine agonists, Cabergoline, Bromocriptine

\section{Introducción}

El prolactinoma constituye el $40 \%$ de los tumores hipofisarios funcionales. La hiperprolactinemia secundaria, puede ser asintomática o resultar en hipogonadismo, infertilidad, galactorrea y pérdida de hasta el $25 \%$ de la densidad mineral ósea en mujeres ${ }^{(1)}$.

La prevalencia varía entre 6 y 10 por 100.000 , con un pico en mujeres con edades entre 25 y 34 años $^{(1)}$. En Colombia, Reyes (1981) describió una serie de nueve casos de pacien- 
tes con prolactinoma tratados de forma exitosa con $\mathrm{BRC}^{(2)}$. La revisión de diferentes bases de datos no reportó información acerca de su prevalencia en Colombia.

Los principales agentes farmacológicos disponibles en nuestro medio son los agonistas de dopamina derivados del ergot: BRC y $\mathrm{CAB}^{(3)}$. La BRC ha sido utilizada desde 1970 , es efectiva en 60 a $80 \%$ de los microprolactinomas y 50 a $70 \%$ de los macroprolactinomas ${ }^{(4)}$. La CAB se encuentra disponible desde hace más de 15 años, es un agonista selectivo del receptor D2, a dosis bajas durante 6 a 24 meses, normaliza niveles de PRL y disminuye el tamaño tumoral hasta en el $90 \%$ de los pacientes, con mínimos efectos adversos ${ }^{(5 ; 6)}$. La cirugía se considera terapia de segunda línea en pacientes resistentes al tratamiento médico (tabla 1) ${ }^{(7)}$. Aunque el prolactinoma es el tumor menos radiosensible comparado con otros tumores funcionales hipofisarios, con tasas de remisión del $18 \%$ a 4 años, la radioterapia se considera tercera opción en pacientes que no responden adecuadamente a las líneas de tratamiento previas $^{(5 ; 8)}$.

El objetivo de este estudio es describir la experiencia del servicio de endocrinología del Hospital San José de Bogotá en el manejo del adenoma hipofisario productor de PRL.

\section{Métodos}

Diseño: Se realizó una serie de casos. Ingresaron pacientes que consultaron al servicio de endocrinología del Hospital San José, desde enero de 2006 hasta diciembre de 2012, con lesión tumoral hipofisaria documentada por RNM contrastada, reportada en historia clínica, y PRL sérica mayor de 100 $\mathrm{ng} / \mathrm{ml}$, o remitidos de otras instituciones con diagnóstico de prolactinoma.

Se excluyeron pacientes en gestación o lactancia, hipotiroidismo primario no controlado, hiperprolactinemia secundaria a medicamentos, desviación del tallo hipofisario y adenoma hipofisario productor de hormona de crecimiento.

Las variables estudiadas fueron: edad, sexo, síntomas al momento del diagnóstico, antecedentes patológicos, entre ellos: hipotiroidismo (de cualquier etiología), osteoporosis, hipogonadismo y medidas antropométricas. Se registró el tipo de agonista de dopamina utilizado, el tiempo de tratamiento en meses, la dosis acumulada (tabla 1) y la causa de suspensión del mismo. Dentro de las variables radiológicas documentadas en la RNM de silla turca contrastada, de ingreso y de seguimiento se definió: microprolactinoma, adenoma menor a $10 \mathrm{~mm}$; macroprolactinoma, adenoma mayor o igual a 10 $\mathrm{mm}$, y adenoma gigante, mayor a $40 \mathrm{~mm}$; adicionalmente se registró tamaño tumoral, compresión del quiasma óptico y/o infiltración del seno cavernoso.

Se recolectaron los valores registrados en la historia clínica de PRL sérica de ingreso, 6 meses y 24 meses posteriores al inicio de tratamiento. El factor de conversión fue $1 \mathrm{ng} / \mathrm{ml}=21$
Tabla 1. Definiciones

\begin{tabular}{c|l}
\multicolumn{1}{c}{ Tabla 1. Definiciones } \\
\hline \multicolumn{1}{c|}{ Concepto } & \multicolumn{1}{c}{ Definición } \\
\hline Dosis acumulada & $\begin{array}{l}\text { Fue calculada para cada paciente como la } \\
\text { sumatoria de la dosis máxima utilizada en el } \\
\text { mes, multiplicada por el número de meses de } \\
\text { tratamiento. }{ }^{(11)}\end{array}$ \\
\hline Resistencia a BRC & $\begin{array}{l}\text { Falla en normalizar los niveles de PRL a pesar } \\
\text { de administrar 15 mg/día de BRC durante tres } \\
\text { meses. }{ }^{(4 ; 8)}\end{array}$ \\
\hline Resistencia a CAB & $\begin{array}{l}\text { Falla en normalizar los niveles de PRL }{ }^{(6)} \mathrm{y} / \mathrm{o} \text { dis- } \\
\text { minución del tamaño tumoral menor al } 50 \% \\
\text { a pesar de administrar } 2.0 \mathrm{mg} / \mathrm{semanales} \mathrm{de} \\
\text { CAB. }{ }^{(8)}\end{array}$ \\
\hline
\end{tabular}

$\mathrm{mUI} / \mathrm{l}^{(1)}$. Se registró el tiempo de seguimiento, la presencia de embarazo durante el tratamiento, los hallazgos en campimetría, densitometría ósea y ecocardiograma de los pacientes de quienes se disponía esta información.

Se consideró resistencia a $\mathrm{CAB}$ en aquellos pacientes en quienes no se logró normalizar los niveles de PRL o la disminución del tamaño tumoral fue menor al $50 \%$ a pesar de administrar dosis $\geq 2,0 \mathrm{mg} /$ semanales (tabla 1).

\section{Consideraciones éticas}

El estudio fue aprobado por el comité de investigación y ética de la Facultad de Medicina de la Fundación Universitaria de Ciencias de la Salud, Hospital San José de Bogotá.

\section{Análisis estadístico}

Se realizó una descripción de las variables cuantitativas a través de medidas de tendencia central y dispersión; las variables cualitativas se describen por medio de proporciones. El análisis de los datos se realizó con el programa STATA $10^{\circledR}$.

\section{Resultados}

En el servicio de endocrinología del Hospital San José de Bogotá, se registraron 980 consultas en 215 pacientes con tumores hipofisarios en el periodo comprendido entre enero de 2006 y diciembre de 2012, de estos, 99 casos correspondían a prolactinoma. Se analizaron las características basales de 95 pacientes, se excluyeron 4; 2 por datos incompletos, 1 por gestación y porque no siguió controles en el servicio, y 1 con inmunohistoquímica compatible con craneofaringioma (figura 1).

En la tabla 2 se describen las características de la población. El promedio de edad fue 36,3 $\pm 10,6$ años, el $83 \%$ fueron mujeres. Los síntomas más frecuentes de consulta fueron galactorrea, amenorrea y cefalea; el 6,4\% consultó por infertilidad. Durante el seguimiento, la alteración del perfil hipofisario más frecuente fue el hipogonadismo hipogonadotrófico, segui- 


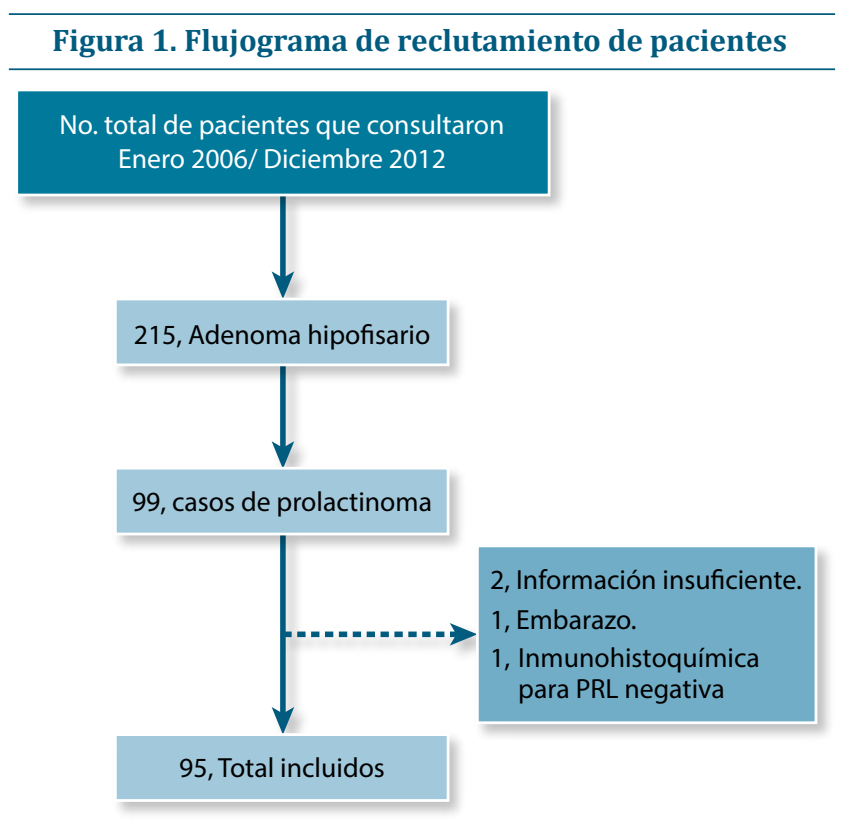

do por el hipotiroidismo central (tabla 3). A pesar de que el prolactinoma constituye el principal tipo histológico de tumor hipofisario en NEM 1 (neoplasia endocrina múltiple) ${ }^{(9 ; 10)}$, no se documentó ninguno en nuestra serie de casos.

La mediana de PRL al ingreso de los pacientes fue de 111 ng/ml (65,3 - 200), durante el seguimiento que se realizó en el servicio de endocrinología se observó una diferencia de medianas de $60,1 \mathrm{ng} / \mathrm{ml}$ con respecto a la medición registrada a los 6 meses y de 78,2 ng/ml con relación a la PRL observada a los 24 meses (figura 2).

El 71\% de los pacientes se clasificaron como microprolactinomas, de ellos el $66 \%$ son mujeres, con edad promedio de diagnóstico 28,7 \pm 9,2 años. El 28,4\% se clasificó como macroprolactinomas, con edad promedio de diagnóstico de 34,9 \pm 13 años; de éstos, el 29,6\% presentó compromiso quiasmático y/o invasión a senos cavernosos. En el seguimiento radiológico se documentó desaparición de la lesión en 42,2\% de los microprolactinomas, comparado con $18,2 \%$ de los pacientes con macroprolactinomas.

Tabla 2. Características basales de la población según clasificación por tamaño tumoral

\begin{tabular}{l|r|l|r|l|r|r}
\hline & Microprolactinoma & Macroprolactinoma* & \multicolumn{2}{|c|}{ Total } \\
\hline Edad, años, promedio (DE) & 68 & $(71,6)$ & 27 & $(28,4)$ & 95 & $(100)$ \\
\hline Mínima - Máxima & 35,1 & $(9,4)$ & 39,2 & $(12,8)$ & 36,3 & $(10,6)$ \\
\hline Edad al diagnóstico, promedio (DE) & $16-61$ & $16-$ & 65 & $16-$ & 65 \\
\hline
\end{tabular}

Sexo, $n(\%)$

\begin{tabular}{|c|c|c|c|c|c|c|}
\hline Femenino & 66 & (97) & 17 & (63) & 83 & $(87,4)$ \\
\hline \multicolumn{7}{|l|}{ Síntomas en el momento del diagnóstico ${ }^{+}$} \\
\hline Galactorrea & 40 & $(59,7)$ & 10 & $(38,5)$ & 50 & $(53,8)$ \\
\hline Amenorrea & 36 & $(53,7)$ & 12 & $(46,1)$ & 48 & $(51,6)$ \\
\hline Oligomenorrea & 17 & $(25,4)$ & 2 & $(7,4)$ & 19 & $(20,4)$ \\
\hline Cefalea & 35 & $(52,2)$ & 15 & $(57,7)$ & 50 & $(53,8)$ \\
\hline Síntomas visuales ${ }^{\ddagger}$ & 7 & $(10,4)$ & 11 & $(42,3)$ & 18 & $(19,3)$ \\
\hline Disfunción eréctil & 1 & $(1,5)$ & 2 & $(7,7)$ & 3 & $(3,2)$ \\
\hline Disminución de libido & 5 & $(7,5)$ & 5 & $(18,5)$ & 10 & $(10,7)$ \\
\hline Aumento de peso & 4 & $(6,0)$ & 0 & & 2 & $(2,1)$ \\
\hline Paridad insatisfecha & 6 & $(8,9)$ & 0 & & 6 & $(6,4)$ \\
\hline \multicolumn{7}{|l|}{ Comorbilidades, $n$ (\%) } \\
\hline Hipotiroidismo primario controlado" & 9 & $(13,2)$ & 4 & $(14,8)$ & 14 & $(14,7)$ \\
\hline
\end{tabular}

* En el grupo de macroprolactinoma se incluyeron 4 pacientes con adenoma hipofisario gigante. ${ }^{\dagger}$ Datos disponibles para: 67 pacientes del grupo de microprolactinoma, 26 pacientes del grupo de macroprolactinoma y 93 pacientes en el grupo total. `Disminución de agudeza visual en 4 pacientes; 1 paciente presentó defecto del campo visual. ${ }^{\$} 7$ pacientes con defecto de campo visual, 1 paciente con diplopía, 3 pacientes con disminución de la agudeza visual. ${ }^{\circledR S e}$ incluyó 1 paciente con hipotiroidismo secundario a tiroidectomía total, con patología benigna. 
Tabla 3. Seguimiento de pacientes con adenoma hipofisario productor de PRL según clasificación por tamaño tumoral

\begin{tabular}{|c|c|c|c|c|c|c|}
\hline & \multicolumn{2}{|c|}{ Microprolactinoma } & \multicolumn{2}{|c|}{ Macroprolactinoma* } & \multicolumn{2}{|c|}{ Total } \\
\hline & 68 & $(71,6)$ & 27 & $(28,4)$ & 95 & $(100)$ \\
\hline \multicolumn{7}{|l|}{$P R L$ al ingreso $\mathrm{ng} / \mathrm{ml}^{+}$} \\
\hline Promedio (DS) & 173 & $(224,4)$ & 806,1 & $(1952,4)$ & 354,9 & (1087) \\
\hline Mediana (RIQ) & 109,5 & $(73,4-160)$ & 111,6 & $(54,5-470)$ & 111 & $(65,3-200)$ \\
\hline \multicolumn{7}{|l|}{$P R L 6$ meses $n g / m^{*}$} \\
\hline Promedio (DS) & 124,8 & $(272,9)$ & 284,2 & $(402,5)$ & 173,3 & $(321,9)$ \\
\hline Mediana (RIQ) & 35,6 & $(14,5-92,2)$ & 92,8 & $(25,6-470)$ & 50,9 & $(15,9-100,1)$ \\
\hline \multicolumn{7}{|l|}{ PRL 24 meses, $\mathrm{ng} / \mathrm{m}^{\S}$} \\
\hline Promedio (DS) & 58,3 & $(67,7)$ & 39,5 & $(78,0)$ & 53,3 & $(70,4)$ \\
\hline Mediana (RIQ) & 41,7 & $(18,1-59,1)$ & 10,22 & $(4,2-24,9)$ & 32,8 & $(15,5-56,1)$ \\
\hline Número de mediciones de PRL, promedio (DS) & 4,3 & $(3,4)$ & 4,8 & $(4,7)$ & 4,4 & $(3,7)$ \\
\hline \multicolumn{7}{|l|}{ Tamaño tumoral en RNM de silla turca inicial, mm" } \\
\hline Promedio (DS) & 5,5 & $(2,4)$ & 20,8 & $(16,3)$ & 10,7 & $(12,0)$ \\
\hline Mediana (RIQ) & 5,4 & $(4-7)$ & 14,5 & $(10-30)$ & 7 & $(5-10)$ \\
\hline Invasión seno cavernoso, n(\%) & 0 & & 8 & $(29,6)$ & 8 & $(8,4)$ \\
\hline Invasión quiasma óptico, n(\%) & 0 & & 8 & $(29,6)$ & 8 & $(8,4)$ \\
\hline \multicolumn{7}{|c|}{ Tamaño tumoral última RNM silla turca contrastada, $\mathrm{mm}^{* *}$} \\
\hline Promedio (DS) & 3,3 & $(3,4)$ & 10,1 & $(11,7)$ & 5,4 & $(7,6)$ \\
\hline Mediana (RIQ) & 3 & $(0-6)$ & 8 & $(2-13,5)$ & 4 & $(0-8,2)$ \\
\hline Desaparición de la lesión hipofisaria, n(\%) & 16 & $(42,2)$ & 2 & $(18,2)$ & 18 & $(33,9)$ \\
\hline Número de RNM de silla turca, promedio (DS) & 2,2 & $(1,1)$ & 1,9 & $(1,0)$ & 2 & $(1,0)$ \\
\hline Embarazo, n(\%) & 6 & $(8,8)$ & 0 & & 6 & $(6,3)$ \\
\hline Seguimiento en años, promedio (DS) & 4,7 & $(4,4)$ & 4,7 & $(4,3)$ & 4,7 & $(4,3)$ \\
\hline Perfil hipofisario, $n$ (\%) & 20 & $(29,4)$ & 13 & $(48,1)$ & 33 & $(34,7)$ \\
\hline Hipotiroidismo central & 0 & & 6 & $(22,2)$ & 6 & $(6,3)$ \\
\hline Hipogonadismo central & 3 & $(4,41)$ & 10 & (37) & 13 & $(13,7)$ \\
\hline Insuficiencia adrenal central & 0 & & 2 & $(7,4)$ & 2 & $(2,1)$ \\
\hline Campimetría, n (\%) & 8 & $(11,8)$ & 12 & $(44,4)$ & 20 & (21) \\
\hline Hallazgos anormales & 3 & $(4,44)$ & 5 & $(18,5)$ & 8 & $(8,4)$ \\
\hline Ecocardiograma, normal/anormal & 3 & 10 & 9 & $/ 3$ & 12 & $/ 3$ \\
\hline
\end{tabular}

* En el grupo de macroprolactinoma se incluyeron 4 pacientes con adenoma hipofisario gigante. ${ }^{\dagger}$ Datos disponibles de 87 pacientes. ${ }^{\ddagger}$ Datos disponibles de 46 pacientes. ${ }^{\S}$ Datos disponibles de 56 pacientes. ${ }^{~ D a t o s ~ d i s p o n i b l e s ~ d e ~} 65$ pacientes.**Datos disponibles de 53 pacientes. ${ }^{+\dagger}$ Datos disponibles de 75 pacientes.(DS): Desviación estándar, (RIQ): Rango intercuartílico.

De los 27 pacientes con antecedente de macroprolactinoma se reportaron 12 campimetrías, 5 de ellas anormales; sin embargo, sólo uno de estos hallazgos se relacionó con efecto compresivo del quiasma óptico; de 9 ecocardiogramas, se reportaron 3 anormales, ninguno de éstos reportó alteración valvular. En 4 de 7 densitometrías óseas se encontró densidad mineral ósea baja para la edad (tabla 3).

La principal indicación de tratamiento médico fue el control de los síntomas relacionados con hiperprolactinemia. El $78,7 \%$ de los pacientes recibió tratamiento con BRC, mediana de dosis acumulada de 2.737,5 (1.350 - 6.159,4) mg, la mediana de duración de tratamiento fue de 30,2 (12,2 - 57,2) meses.
El $64,9 \%$ de los pacientes recibió tratamiento con $\mathrm{CAB}$, con dosis acumulada de 123,6 $\pm 146,8 \mathrm{mg}$, la mediana de duración del tratamiento fue de 48,7 meses (tabla 4). El 9,5\% de los pacientes fueron tratados con cirugía. Ningún paciente requirió radioterapia.

La principal causa de interrupción de tratamiento estuvo relacionada con eventos adversos gastrointestinales (tabla 5), más frecuentes en el grupo tratado con BRC. Ocho pacientes cumplieron criterios de remisión. La resistencia a la BRC se registró en un paciente con macroprolactinoma, el cual respondió adecuadamente al tratamiento con CAB. Se documentó resistencia a la $\mathrm{CAB}$ en dos pacientes masculinos. 
Figura 2. Seguimiento bioquímico con PRL basal, 6 y 24 meses. En el recuadro 1 se presentan los niveles de prolactina de los microadenomas y en el recuadro 2 el de los macroadenomas. En esta gráfica de boxplot, se excluyeron valores extremos de PRL
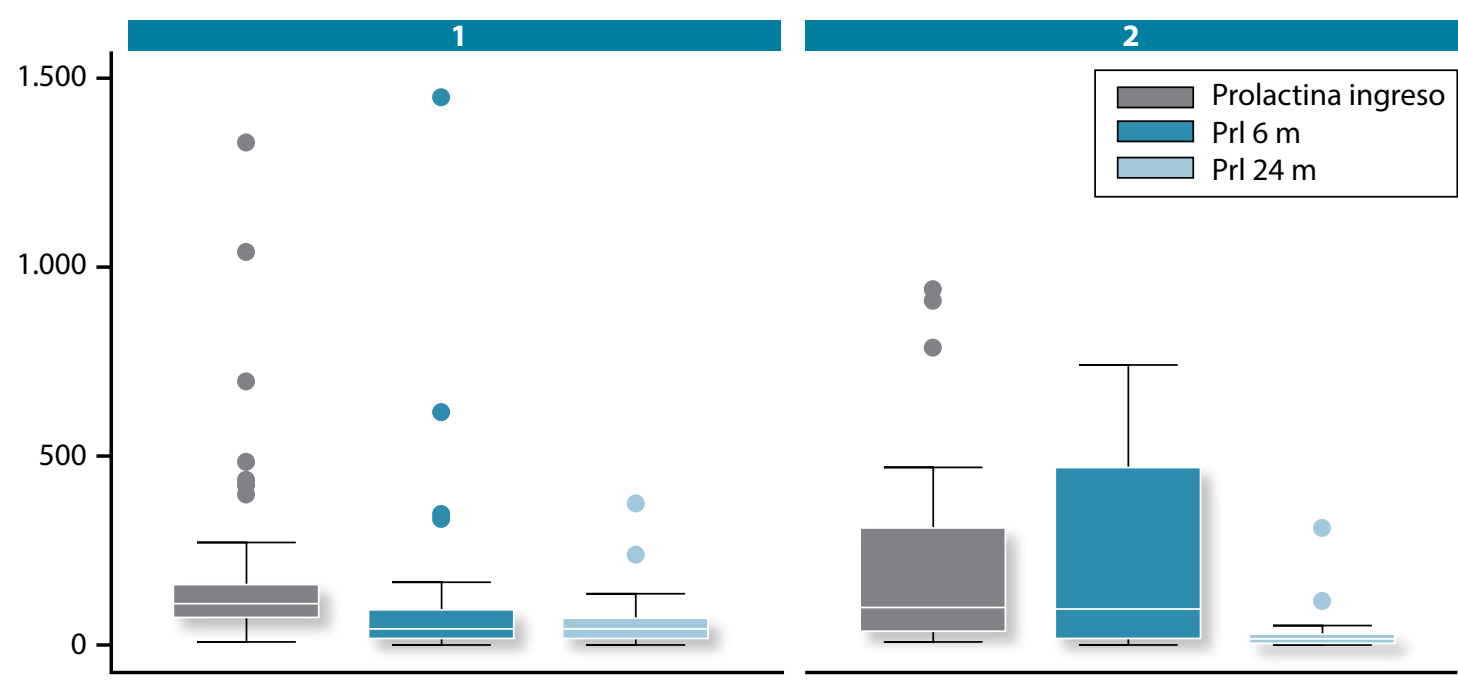

Tabla 4. Tratamiento de adenoma hipofisario productor de PRL, según su clasificación por tamaño tumoral

\begin{tabular}{|c|c|c|c|c|c|c|}
\hline & \multicolumn{2}{|c|}{ Microprolactinoma* } & \multicolumn{2}{|c|}{ Macroprolactinoma $^{\dagger}$} & \multicolumn{2}{|c|}{ Total ${ }^{\ddagger}$} \\
\hline & 68 & $(71,6)$ & 27 & $(28,4)$ & 95 & $(100)$ \\
\hline \multicolumn{7}{|l|}{ Tipo de agonista de dopamina, $n$ (\%) } \\
\hline BRC & 57 & (85) & 17 & (63) & 74 & $(78,7)$ \\
\hline CAB & 42 & $(62,7)$ & 19 & $(70,4)$ & 61 & $(64,9)$ \\
\hline Terapia con $>1$ agonista de dopamina & 32 & $(47,8)$ & 9 & $(33,3)$ & 41 & $(43,6)$ \\
\hline \multicolumn{7}{|l|}{ Dosis, mediana (RIQ) } \\
\hline BRC, mg/día & 2,5 & $(2,5-5)$ & 5 & $(2,5-7,5)$ & 2,5 & $(2,5-5)$ \\
\hline $\mathrm{CAB}, \mathrm{mg} / \mathrm{semana}$ & 0,5 & $(0,5-0,5)$ & 0,5 & $(0,5-1,5)$ & 0,5 & $(0,5-1,0)$ \\
\hline \multicolumn{7}{|l|}{ Dosis acumulada mediana, $\mathrm{mg}$} \\
\hline BRC, promedio (DS) & $5.174,9$ & $(5509,7)$ & $7.481,6$ & $(19342,5)$ & $5.704,9$ & $(10.307,5)$ \\
\hline BRC, mediana (RIQ) & 3.650 & $(1.350-7.200)$ & 2.700 & $(912,5-4.562,5)$ & $2.737,5$ & $(1.350-6.159,4)$ \\
\hline CAB, promedio (DS) & 88,5 & $(94,7)$ & 201,1 & $(195,6)$ & 123,6 & $(146,8)$ \\
\hline CAB, mediana (RIQ) & 52 & $(26-104)$ & 156 & $(40-286)$ & 78 & $(26-156)$ \\
\hline Duración de tratamiento, Mediana (RIQ) & 73,4 & $(24,3-122,5)$ & 65 & $(26,8-100,7)$ & 73 & $(24,3-121,7)$ \\
\hline $\mathrm{BRC}$, meses & 36,5 & $(12,2-60)$ & 12,2 & $(12,2-36,5)$ & 30,2 & $(12,2-57,2)$ \\
\hline$C A B$, meses & 48,7 & $(17,5-79,8)$ & 49 & $(15,1-86,3)$ & 48,7 & $(17-85,4)$ \\
\hline \multicolumn{7}{|l|}{ Tratamiento quirúrgico, $n(\%)$} \\
\hline Resección transesfenoidal & 1 & $(1,5)$ & 5 & $(18,5)$ & 6 & $(6,3)$ \\
\hline Resección transcraneal & 0 & & 3 & $(11,1)$ & 3 & $(3,2)$ \\
\hline
\end{tabular}

*Datos disponibles de 67 pacientes. ${ }^{\dagger}$ En el grupo de macroprolactinoma se incluyeron 4 pacientes con adenoma hipofisario gigante. ${ }^{\star}$ Datos disponibles de 94 pacientes 
Tabla 5. Causa de suspensión de agonista de dopamina

\begin{tabular}{l|r|l|r|l}
\hline & \multicolumn{2}{|c|}{ BRC } & \multicolumn{2}{|c}{ CAB } \\
\hline Total tratados, $\mathrm{n}(\%)$ & 74 & & 61 & \\
\hline Efectos adversos gastrointestinales* & 26 & $(35)$ & 0 & \\
\hline Cefalea & 1 & $(1,3)$ & 0 & \\
\hline Exantema & 0 & & 1 & $(1,6)$ \\
\hline Embarazo & 3 & $(4)$ & 3 & $(4,9)$ \\
\hline Pobre adherencia al tratamiento + & 2 & $(2,7)$ & 1 & $(1,6)$ \\
\hline Retiro & 3 & $(4)$ & 5 & $(6,7)$ \\
\hline Sin registro & 4 & $(5,4)$ & 1 & $(1,6)$ \\
\hline Resistencia $\neq$ & 1 & $(1,3)$ & 2 & $(3,2)$ \\
\hline
\end{tabular}

*Eventos adversos gastrointestinales: síntomas como náuseas, vómito, diarrea o dolor abdominal, por los cuales se suspendió el tratamiento. †Pobre adherencia: suspensión de medicamento por voluntad propia. ${ }^{\ddagger}$ Resistencia: según definición descrita en la tabla 1.

\section{Discusión}

El prolactinoma es el tumor hipofisario funcionante más frecuente. Representa entre 50 y $66 \%$ de todos los tumores secretores hipofisarios, aunque no hay datos en Colombia, en el servicio de endocrinología del Hospital San José de Bogotá, $37 \%$ de los tumores hipofisarios pertenecen al grupo de adenomas hipofisarios productores de PRL.

De los pacientes con microprolactinomas, encontramos que las características de nuestra población, la edad de presentación, síntomas y relación masculino:femenino fueron similares al comparar con otras series ${ }^{(5 ; 11-15)}$. Sin embargo, el $63 \%$ de los pacientes con macroprolactinomas fueron mujeres, en quienes los síntomas predominantes fueron secundarios a efecto de masa, e hipopituitarismo a diferencia de otras series donde tal presentación es más frecuentemente en hombres con una relación $5: 1^{(16)}$.

El control de síntomas fue la principal indicación de tratamiento con agonistas de dopamina. La duración del tratamiento fue similar a la reportada por la literatura (tabla 6); sin embargo, las dosis requeridas de $\mathrm{CAB}$ en el manejo de pacientes con macroprolactinomas fueron menores en esta serie $(0,5 \mathrm{a}$ $1,5 \mathrm{mg} / \mathrm{semana}$ ) al comparar con estudios prospectivos donde el promedio de dosis fue de 0,25 a 2,0 mg/semana, logrando normalización de niveles de PRL en el $81 \%$ y disminución de tamaño tumoral en el $91 \%$ a los 6 meses de seguimiento; en pacientes masculinos la dosis promedio fue de 2,5 a $3,5 \mathrm{mg} /$ semanal, logrando normalización de PRL en el 76\% luego de 24 meses de seguimiento ${ }^{(16)}$.

Ningún estudio ha encontrado relación entre factores como el tamaño tumoral, niveles de PRL antes del retiro de los agonistas de dopamina y la tasa de recurrencia ${ }^{(5 ; 17)}$. Existen reportes de tasas de remisión hasta de $70 \%$ en microprolactinomas y de $45 \%$ en macroprolactinomas, y los estudios que han descrito las tasas más bajas reportan el $21 \%{ }^{(12)}$. En la serie, 26 pacientes normalizaron sus niveles de PRL, en 18 pacientes la lesión desapareció y 66 pacientes completaron tratamiento con agonistas de dopamina por un periodo mayor o igual a dos años y sólo se logró retiro exitoso en el 8,4\% de pacientes, hallazgos que se pueden atribuir a:

- Las tasas altas de remisión se observan en series con uso exclusivo de $\mathrm{CAB}^{(12)}$, mientras que en esta serie el inicio de tratamiento fue para la mayoría de pacientes con BRC.

- Los estudios que reportan tasas de remisión informan un porcentaje de cambio de BRC a CAB mayor a $57 \%{ }^{(13 ; 14)}$. En esta serie fue de $43,2 \%$.

- En esta investigación la principal limitación para el ajuste de dosis fue la intolerancia gastrointestinal a la $\mathrm{BRC}$, la cual fue mayor comparada con otros estudios (35\% vs $12 \%)^{(4)}$.

- La duración promedio de tratamiento con BRC fue similar a la de otras series, sin embargo, la dosis acumulada fue menor y se observó el mismo fenómeno con la $\mathrm{CAB}$ (tabla 6). Por lo cual, el uso de dosis acumuladas más bajas podría ser otro factor asociado a la baja tasa de remisión.

Según Oh, 2011, el 10\% de los pacientes con prolactinomas son resistentes a los agonistas de dopamina ${ }^{(8)}$. En este estudio, el reporte de resistencia a BRC y CAB fue menor al informado en la literatura mundial. En estudios retrospectivos,

Tabla 6. Comparación de seguimiento

\begin{tabular}{|c|c|c|c|c|c|c|c|c|c|c|c|c|c|}
\hline \multirow{2}{*}{$\begin{array}{c}\begin{array}{c}\text { Primer autor, } \\
\text { año [Ref.] }\end{array} \\
\text { Bogazzi, 2008(15) }\end{array}$} & \multicolumn{2}{|c|}{$\begin{array}{c}\text { No. de } \\
\text { pacientes con } \\
\text { BRC/No. total } \\
\text { de pacientes }\end{array}$} & \multicolumn{2}{|c|}{ Sexo (M/F) } & \multicolumn{2}{|c|}{$\begin{array}{l}\text { Edad (años) } \\
\text { (Prom } \pm \text { SD) }\end{array}$} & \multirow{2}{*}{$\begin{array}{c}\begin{array}{c}\text { BRC, Dosis AC } \\
\text { (mg), Prom } \pm \text { SD }\end{array} \\
13670 \pm 17105\end{array}$} & \multicolumn{2}{|c|}{$\begin{array}{l}\text { DT con BRC (meses), } \\
\text { prom } \pm \text { SD (rango) }\end{array}$} & \multicolumn{2}{|c|}{$\begin{array}{c}\text { CAB, Dosis Ac (mg), Prom } \pm \\
\text { SD (rango) }\end{array}$} & \multicolumn{2}{|c|}{$\begin{array}{l}\text { DT con CAB (meses), } \\
\text { prom } \pm \text { SD (rango) }\end{array}$} \\
\hline & 21 & $/ 100$ & 21 & 179 & 41 & (13) & & $61 \pm 43$ & $(6-169)$ & $279 \pm 301$ & $(15-1327)$ & $67 \pm 39$ & (3-199) \\
\hline Kars, $2008^{(13)}$ & 20 & 178 & 13 & $/ 34$ & 47 & (1) & $4216 \pm 899$ & $34,8 \pm 7,2$ & & $363 \pm 55$ & $(24-1758)$ & $62 \pm 5$ & $(12-124)$ \\
\hline Vallette, $200 g^{(14)}$ & 40 & 170 & 33 & $/ 37$ & 44 & & (ND) & 12 & $(6-120)$ & $282 \pm 271$ & & $55 \pm 22$ & \\
\hline Colao, $2007^{(12)}$ & 0 & 150 & 6 & 144 & 36 & $(10)$ & & & & $414 \pm 390$ & $(32-1938)$ & 74 & $(16-260)$ \\
\hline HSJ, 2012 & 74 & 195 & 12 & $/ 83$ & 36,3 & $(10,6)$ & $5704,9 \pm 10307,5$ & $42 \pm 49$ & $(1-255,5)$ & $123,6 \pm 146,8$ & $(3-624)$ & $59,7 \pm 53,5$ & $(6,5-294,5)$ \\
\hline
\end{tabular}

M, masculino; F, Femenino; Prom, promedio; SD, desviación estándar; BRC, bromocriptina, CAB, cabergolina; Dosis Ac, dosis acumulada; DT, duración de tratamiento 
la resistencia a agonistas de dopamina se ha relacionado con características histológicas como el índice de proliferación y atipia celular, así como la invasión al seno cavernoso y el sexo masculino ${ }^{(17)}$. Estos últimos fueron hallazgos poco frecuentes en la población de estudio, sin embargo, los pacientes con resistencia a BRC y CAB en esta serie eran hombres con adenomas gigantes.

Los resultados del estudio son relevantes porque en Colombia existen pocas investigaciones para la caracterización demográfica de la población con diagnóstico de adenoma hipofisario productor de PRL. A pesar de que existen reportes de casos de tratamiento médico, en ellos no se llevó a cabo seguimiento bioquímico ni radiológico. Los resultados obtenidos permiten la identificación de potenciales variables que podrían influir en la duración del tratamiento y el retiro exitoso del mismo. Por ser un estudio retrospectivo, la recolección de los datos dependió de la calidad del registro en la historia clínica.

Se deben realizar estudios prospectivos para aclarar si la dosis acumulada es un factor predictor para diseñar estrategias que permitan aumentar el porcentaje de pacientes con retiro exitoso de agonistas de dopamina en pacientes con prolactinomas.

\section{Conclusión}

El prolactinoma es el tumor hipofisario funcional más frecuente. En la población del Hospital San José se identificaron características similares a las reportadas en la literatura, incluyendo esquema de tratamiento. A pesar de esto, durante el seguimiento el porcentaje de remisión en la población estudiada es bajo. Se requieren estudios prospectivos para aclarar si la dosis acumulada es un factor predictor para aumentar el porcentaje de pacientes con retiro exitoso y establecer la mejor estrategia para retiro de agonistas de dopamina en pacientes con prolactinomas.

\section{Referencias}

1. Melmed S, Casanueva FF, Hoffman AR, Kleinberg DL, Montori VM, Schlechte JA, et al. Diagnosis and treatment of hyperprolactinemia: an Endocrine Society clinical practice guideline. J Clin Endocrinol Metab 2011;96(2):273-88.

2. Reyes Leal B. Prolactinoma: tratamiento médico. Acta Medica Colombiana [6], 217-223. 1981.

3. Klibanski A. Clinical practice. Prolactinomas. N Engl J Med 2010; ;362(13):1219-26.

4. Hamilton DK, Vance ML, Boulos PT, Laws ER. Surgical outcomes in hyporesponsive prolactinomas: analysis of patients with resistance or intolerance to dopamine agonists. Pituitary 2005;8(1):53-60.
5. Antagnostis P, Adamidou F, Polyzos SA, Efstathiadou Z, Karathanassi E, Kita M. Long term follow-up of patients with prolactinomas and outcome of dopamine agonist withdrawal: a single center experience. Pituitary 2012 Mar;15(1):25-9.

6. Vroonen L, Jaffrain-Rea ML, Petrossians P, Tamagno G, Chanson P, Vilar L, et al. Prolactinomas resistant to standard doses of cabergoline: a multicenter study of 92 patients. Eur J Endocrinol 2012;167(5):651-62.

7. Hamilton DK, Vance ML, Boulos PT, Laws ER. Surgical outcomes in hyporesponsive prolactinomas: analysis of patients with resistance or intolerance to dopamine agonists. Pituitary 2005;8(1):53-60.

8. Oh MC, Aghi MK. Dopamine agonist-resistant prolactinomas. J Neurosurg 2011 May;114(5):1369-79.

9. Colao A. Pituitary tumours: the prolactinoma. Best Pract Res Clin Endocrinol Metab 2009;23(5):575-96.

10. Fajardo-Montanana C, Daly AF, Riesgo-Suarez P, GomezVela J, Tichomirowa MA, Camara-Gomez R, et al. [AIP mutations in familial and sporadic pituitary adenomas: local experience and review of the literature]. Endocrinol Nutr 2009;56(7):369-77.

11. Lancellotti P, Livadariu E, Markov M, Daly AF, Burlacu MC, Betea D, et al. Cabergoline and the risk of valvular lesions in endocrine disease. Eur J Endocrinol 2008;159(1):1-5.

12. Colao A, Di SA, Guerra E, Pivonello R, Cappabianca P, Caranci F, et al. Predictors of remission of hyperprolactinaemia after long-term withdrawal of cabergoline therapy. Clin Endocrinol (Oxf) 2007;67(3):426-33.

13. Kars M, Delgado V, Holman ER, Feelders RA, Smit JW, Romijn JA, et al. Aortic valve calcification and mild tricuspid regurgitation but no clinical heart disease after 8 years of dopamine agonist therapy for prolactinoma. J Clin Endocrinol Metab 2008;93(9):3348-56.

14. Vallette S, Serri K, Rivera J, Santagata P, Delorme S, Garfield $\mathrm{N}$, et al. Long-term cabergoline therapy is not associated with valvular heart disease in patients with prolactinomas. Pituitary 2009;12(3):153-7.

15. Bogazzi F, Buralli S, Manetti L, Raffaelli V, Cigni T, Lombardi $\mathrm{M}$, et al. Treatment with low doses of cabergoline is not associated with increased prevalence of cardiac valve regurgitation in patients with hyperprolactinaemia. Int J Clin Pract 2008 Dec;62(12):1864-9.

16. Iglesias P, Diez JJ. Macroprolactinoma: a diagnostic and therapeutic update. QJM 2013 Jun;106(6):495-504.

17. Delgrange E, Sassolas G, Perrin G, Jan M, Trouillas J. Clinical and histological correlations in prolactinomas, with special reference to bromocriptine resistance. Acta Neurochir (Wien ) 2005;147(7):751-7. 